\title{
PREVENÇÃO DE ÚLCERAS POR PRESSÃO: AVALIAÇÃO DO CONHECIMENTO DOS PROFISSIONAIS DE ENFERMAGEM
}

\author{
Layrianne Emmanuely Silva Rocha ${ }^{1}$, Edna de Freitas Gomes Ruas², Jaciara Aparecida Dias Santos ${ }^{3}$, Cássio de Almeida Lima ${ }^{4}$,
} Jair Almeida Carneiro ${ }^{5}$, Fernanda Marques da Costa ${ }^{6}$

${ }^{1}$ Enfermeira. Especialista em Saúde da Família. Secretaria Municipal de Saúde. Jaíba, MG, Brasil.

${ }^{2}$ Enfermeira. Mestre em Ciências da Saúde. Docente do Departamento de Enfermagem da Universidade Estadual de Montes Claros. Montes Claros, MG, Brasil.

${ }^{3}$ Acadêmica do Curso de Graduação em Medicina. Universidade Estadual de Montes Claros. Montes Claros, MG, Brasil.

${ }^{4}$ Enfermeiro. Mestrando em Saúde, Sociedade e Ambiente. Universidade Federal dos Vales do Jequitinhonha e Mucuri. Diamantina, MG, Brasil.

${ }^{5}$ Médico. Doutorando em Ciências da Saúde. Docente das Faculdades Integradas Pitágoras de Montes Claros. Montes Claros, MG, Brasil.

${ }^{6}$ Enfermeira. Doutoranda em Ciências da Saúde. Docente das Faculdades Integradas Pitágoras de Montes Claros. Montes Claros, MG, Brasil.

RESUMO: Objetivou-se avaliar o conhecimento da equipe de enfermagem sobre medidas de prevenção de úlceras por pressão em adultos e idosos. Pesquisa descritivo-exploratória e de abordagem quantitativa. Foi utilizado questionário estruturado, a partir do teste de conhecimento de Pieper. A coleta de dados ocorreu entre outubro e dezembro de 2013. O software Statistical Package for the Social Sciences versão 18.0 subsidiou a análise descritiva e bivariada dos dados. Entre os 85 profissionais, a média global do teste aplicado foi $84,21 \%$ para os enfermeiros e de $68,42 \%$ para os técnicos de enfermagem. Apenas $22 \%$ dos enfermeiros acertaram mais que $90 \%$ do teste de conhecimento e apenas $12 \%$ dos técnicos atingiram essa nível. Houve diferença estatisticamente significante entre o maior tempo de serviço, maior idade e categoria profissional, indicando maior conhecimento, sobretudo pelos enfermeiros em alguns aspectos. Há deficiência de conhecimento, necessitando de intervenções educativas constantes e efetivas, a fim de melhorar a assistência.

DESCRITORES: Úlcera por pressão; Prevenção de doenças; Cuidados de enfermagem.

\section{PREVENTION OF PRESSURE ULCERS: EVALUATION OF NURSING PROFESSIONALS' KNOWLEDGE}

ABSTRACT: The aim was to assess the nursing team's knowledge regarding measures for preventing pressure ulcers in adults and older adults. The research was descriptive-exploratory with a quantitative approach. A structured questionnaire was used, based on the Pieper Pressure Ulcer Knowledge test. Data collection took place in October - December 2013. The descriptive and bivariate analysis of the data was undertaken with the support of the Statistical Package for the Social Sciences software, version 18.0. Among the 85 professionals, the global mean of the test applied was $84.21 \%$ for the nurses, and $68.42 \%$ for the nursing technicians. Only $22 \%$ of the nurses obtained correct answers for more than $90 \%$ of the test on their knowledge and only $12 \%$ of the technicians reached this level. There was a statistically significant difference between greater length of service, greater age and professional category, indicating greater knowledge, above all on the part of the nurses in certain aspects. There is a shortage of knowledge, requiring constant and effective educational interventions so as to improve the care provided.

DESCRIPTORS: Pressure ulcer; Prevention of diseases; Nursing care.

\section{PREVENCIÓN DE ÚLCERAS POR PRESIÓN: EVALUACIÓN DEL CONOCIMIENTO DE LOS PROFESIONALES DE ENFERMERÍA}

RESUMEN: El objetivo fue evaluar el conocimiento del equipo de enfermería sobre medidas de prevención de úlceras por presión en adultos y ancianos. Investigación descriptivo exploratoria y de abordaje cuantitativo. Fue utilizado cuestionario estructurado, con test de conocimiento de Pieper. Los datos fueron obtenidos entre octubre y diciembre de 2013. El software Statistical Package for the Social Sciences versión 18.0 subsidió el análisis descriptivo y bivariado de los datos. Entre los 85 profesionales, la media global del test aplicado fue $84,21 \%$ para los enfermeros y de $68,42 \%$ para los técnicos de enfermería. Solamente $22 \%$ de los enfermeiros tuvieron más aciertos que $90 \%$ del test de conocimiento y solo $12 \%$ de los técnicos llegaron a ese nivel. Hubo diferencia estadística significante entre el mayor tiempo de servicio, mayor edad y categoría profesional, apuntando mayor conocimiento, sobretodo por los enfermeros en algunos aspectos. Hay deficiencia de conocimiento, necesitando de intervenciones educativas constantes y efectivas, a fin de mejorar la asistencia.

DESCRIPTORES: Úlcera por presión; Prevención de enfermedades; Cuidados de enfermería. 


\section{INTRODUÇÃO}

Os avanços médico-científicos, concentrados, sobretudo, em hospitais de nível terciário, levam estes a atender pacientes que necessitam de assistência cada vez mais complexa ${ }^{(1)}$. Quanto mais complexa é a terapêutica exigida, maiores são as chances de desenvolvimento de complicações hospitalares, tais como infecções, erros na administração e preparo das medicações, além de lesões de pele. Nesse panorama, as úlceras por pressão estão entre as lesões mais dispendiosas de uma internação. Cabe, então, às instituições de saúde oferecer serviços assistenciais de qualidade, visando à garantia dos direitos do cliente $^{(2)}$.

Os hospitais universitários estão inseridos nessa realidade e também enfrentam o desafio de oferecer assistência segura e de qualidade à população. Para tanto, devem implantar estratégias de melhorias baseadas em indicadores de qualidade, desenvolvidos de acordo com cada realidade $^{(2)}$. Um indicador importante da qualidade assistencial é a prevalência de úlceras por pressão (UPs), as quais aumentam o período de internação hospitalar e oneram a assistência, pois além de tempo exigem desprendimento financeiro para o tratamento, por vezes demorado. Diante disso, as UPs constituem constantes temas de discussões e preocupações entre os profissionais da área da saúde, sobretudo entre os profissionais de enfermagem ${ }^{(3)}$.

A prevenção das UPs torna-se primordial para a qualidade da assistência prestada, visto que afeta todos os atores envolvidos no processo de cuidar: paciente, família, hospital, instituições de saúde e sociedade como um todo, de forma significante, interferindo na autoimagem e autoestima dos portadores, já que acometem o maior e mais externo órgão do corpo humano ${ }^{(1,4)}$.

Parao sucesso daprevenção,énecessário queos profissionais detenham conhecimento científico suficiente. Existem, em âmbito internacional, inúmeras diretrizes baseadas em evidências, que norteiam as ações de abordagem das UPs. Todavia, no Brasil não há diretriz específica para a prevenção das lesões por pressão, apesar da elevação no número de publicações científicas acerca do assunto ${ }^{(5-6)}$.

A fim de alcançar êxitos na prevenção das UPs, tendo em vista sua natureza multifatorial, é necessário que todos os membros de uma equipe multidisciplinar se esforcem para prevenilas e tratá-las. Entretanto, cabe à equipe de enfermagem a maior parcela do cuidado. Isso porque esta é responsável pelo cuidado direto e pelo gerenciamento da assistência, portanto precisa estar preparada para tal ${ }^{(7-8)}$.

Nesse contexto, o presente estudo justifica-se pela relevância em verificar o conhecimento que a equipe de enfermagem detém sobre a prevenção das UPs, uma vez que a mesma está diretamente relacionada à assistência diária, tanto na prevenção quanto no tratamento. Adicionalmente, poderá contribuir para o avanço do conhecimento nessa área. Dessa forma, o estudo objetivou avaliar o conhecimento da equipe de enfermagem sobre medidas de prevenção de úlceras por pressão em adultos e idosos.

\section{MÉTODO}

Trata-se de uma pesquisa de natureza descritivo-exploratória e abordagem quantitativa. O estudo foi desenvolvido no Hospital Universitário Clemente de Faria (HUCF), situado na cidade de Montes Claros, norte de Minas Gerais (MG). A coleta de dados foi realizada entre os meses de outubro e dezembro de 2013, nos períodos diurno e noturno, após aprovação da instituição cenário.

À época da coleta de dados, o HUCF contava com 104 enfermeiros, 293 técnicos em enfermagem e 91 auxiliares de enfermagem distribuídos pelos diversos setores do hospital. A população foi composta pelos profissionais que atuavam no momento da coleta, nos setores de Clínica Médica (A e B), Clínica Cirúrgica, Unidade de Terapia Intensiva-UTI adulto e Pronto Socorro, após obedecerem aos critérios de inclusão: aceitar participar da pesquisa; estar presente no dia da coleta de dados; prestar assistência direta aos clientes adultos e idosos; ser de faixa etária superior a 18 anos. Foram excluídos do estudo os profissionais que estavam em período de férias ou cumprindo atestado médico; se recusaram a participar da investigação; não foram encontrados para a coleta após de três tentativas.

Foi utilizado um questionário adaptado aos objetivos deste estudo, composto por itens referentes aos dados sociodemográficos e teste de conhecimento, denominado teste de conhecimento de Pieper, validado em estudo anterior realizado no Brasil( ${ }^{(4)}$. O teste visa mensurar o nível de conhecimento dos entrevistados acerca das recomendações para prevenção de UPs. Para confecção do teste, os pesquisadores se basearam em recomendações das diretrizes 
internacionais. O instrumento original contém 41 afirmações classificadas em verdadeiras ou falsas, que contemplam questões sobre avaliação e classificação e medidas preventivas das úlceras por pressão. $\mathrm{O}$ instrumento utilizado neste estudo possui 19 afirmações com questões sobre avaliação, classificação e medidas preventivas de UPs.

O participante respondeu cada questão com julgamento de Verdadeiro (V), Falso (F) ou Não sei (NS). Para cada resposta certa foi atribuído um ponto, para as respostas incorretas ou respondidas com "Não sei" a nota atribuída foi zero. O escore total do teste de conhecimento corresponde à soma de todas as respostas corretas. Para o conhecimento ser considerado adequado, esperava-se que os participantes acertassem $90 \%$ ou mais dos itens no teste, então os escores foram divididos em $<70 \%$, de 70 a $89 \%$ e $>90 \%$ de acertos $^{(4)}$.

A análise considerou os escores dos dois grupos de profissionais: técnicos de enfermagem e enfermeiros. Os dados coletados foram analisados no software Statistical Package for the Social Sciences (SPSS), versão 18.0. Foi processada a análise descritiva, e em seguida a análise bivariada, por meio do teste de qui-quadrado de Pearson. Considerou-se um nível de significância de $5 \%$.

O projeto de pesquisa foi aprovado pelo Comitê de Ética em Pesquisa da Universidade Estadual de Montes Claros (UNIMONTES), por meio do parecer 126.191/2012. Todos os participantes leram e assinaram o Termo de Consentimento Livre e Esclarecido.

\section{RESULTADOS}

Participaram da pesquisa 85 profissionais de enfermagem, entre os quais nove $(10,58 \%)$ eram enfermeiros e $76(89,42 \%)$ eram técnicos de enfermagem, nenhum dos funcionários pertencia à categoria de auxiliar de enfermagem. $\mathrm{Na}$ Tabela 1, estão dispostas as características sociodemográficas dos participantes: 43 (56,9\%) técnicos de enfermagem tinham entre 30-60 anos, $59(77,6 \%)$ eram do sexo feminino e $52(68,4 \%)$ possuíam de 5 a 10 anos de serviço. Quanto aos enfermeiros, sete $(77,8 \%)$ tinha idade menor que 30 anos, seis $(66,7 \%)$ eram do sexo feminino e cinco $(55,6 \%)$ apresentavam tempo de carreira menor que cinco anos.

A Tabela 2 apresenta os acertos obtidos por enfermeiros e técnicos em enfermagem, em cada
Tabela 1 - Características sóciodemográficas dos profissionais de enfermagem. Montes Claros, MG, Brasil, 2013

\begin{tabular}{|c|c|c|c|c|c|c|}
\hline \multirow[t]{2}{*}{$\begin{array}{l}\text { Características } \\
\text { Sóciodemográ- } \\
\text { ficas }\end{array}$} & \multicolumn{2}{|c|}{$\begin{array}{l}\text { Técnicos de } \\
\text { Enfermagem } \\
\quad(n=76)\end{array}$} & \multicolumn{2}{|c|}{$\begin{array}{l}\text { Enfer- } \\
\text { meiros } \\
(n=09)\end{array}$} & \multicolumn{2}{|c|}{$\begin{array}{c}\text { Total } \\
(\mathrm{n}=85)\end{array}$} \\
\hline & $\mathrm{n}$ & $\%$ & $n$ & $\%$ & $\mathrm{n}$ & $\%$ \\
\hline \multicolumn{7}{|l|}{ Idade } \\
\hline$<30$ anos & 30 & 39,5 & 7 & 77,8 & 37 & 43,6 \\
\hline $30-60$ anos & 43 & 56,9 & 2 & 22,2 & 45 & 52,9 \\
\hline Não informada & 3 & 3,9 & 0 & 0 & 3 & 2,5 \\
\hline \multicolumn{7}{|l|}{ Sexo } \\
\hline Feminino & 59 & 77,6 & 6 & 66,7 & 65 & 75,5 \\
\hline Masculino & 17 & 22,4 & 3 & 33,3 & 20 & 24,5 \\
\hline \multicolumn{7}{|l|}{ Tempo de serviço } \\
\hline $\begin{array}{l}\text { Menor que } 5 \\
\text { anos }\end{array}$ & 10 & 13,2 & 5 & 55,6 & 15 & 17,6 \\
\hline 5 a 10 anos & 52 & 68,4 & 4 & 44,4 & 56 & 65,8 \\
\hline 10 a 20 anos & 11 & 14,5 & 0 & 0 & 11 & 12,9 \\
\hline Não informado & 3 & 3,9 & 0 & 0 & 3 & 3,7 \\
\hline
\end{tabular}

questão do teste de conhecimento de Pieper. Os enfermeiros acertaram (78\%) entre 70 a $90 \%$ do teste, enquanto apenas $22 \%$ acertaram mais que $90 \%$, e nenhum obteve menos que $70 \%$ de acerto.

A maioria dos técnicos de enfermagem (88\%) apresentava deficiência no conhecimento sobre as recomendações de prevenção de úlceras, sendo que $5 \%$ deles acertaram menos que $70 \%$ das questões, $83 \%$ acertaram entre 70 e $90 \%$ e apenas $12 \%$ obtiveram mais que $90 \%$ de acertos.

Houve diferença estatisticamente significante $(p<0,05)$ entre o maior tempo de serviço e a maior idade no aumento do conhecimento sobre a prevenção das UPs. Também foi identificada significância entre o conhecimento de enfermeiros e técnicos em enfermagem em relação às questões "Alternância de decúbito de 3/3h", "Alternância de decúbito específico para cada paciente", "Uso de luvas para proteção do calcâneo", "Acamados devem ser movimentados sentados a cada $2 \mathrm{~h}$ ", sendo que os enfermeiros demonstraram mais conhecimento (Tabela 3).

\section{DISCUSSÃO}

As características sociodemográficas dos profissionais desta pesquisa se aproximam das identificadas em estudo realizado em um hospital universitário de Ribeirão Preto, no qual participaram da pesquisa 386 membros da equipe de enfermagem, sendo 250 auxiliares/técnicos 


\begin{tabular}{|c|c|c|c|c|}
\hline \multirow[b]{3}{*}{ Questão } & \multicolumn{4}{|c|}{ Número de Acertos } \\
\hline & \multicolumn{2}{|c|}{ Enfermeiros } & \multicolumn{2}{|c|}{ Técnicos/Auxiliares } \\
\hline & $\begin{array}{l}\text { Frequên- } \\
\text { cia } \\
\text { Absoluta } \\
\quad(\mathbf{n}=9)\end{array}$ & $\begin{array}{l}\text { Frequên- } \\
\text { cia } \\
\text { Relativa } \\
\quad(\%)\end{array}$ & $\begin{array}{l}\text { Frequên- } \\
\text { cia } \\
\text { Absoluta } \\
(\mathbf{n}=85)\end{array}$ & $\begin{array}{l}\text { Frequên- } \\
\text { cia } \\
\text { Relativa } \\
\quad(\%)\end{array}$ \\
\hline $\begin{array}{l}\text { 01. São fatores de risco para o desenvolvimento da úlcera por pressão: } \\
\text { imobilidade, incontinência; nutrição inadequada; e alteração do nível } \\
\text { de consciência. (V) }\end{array}$ & 9 & 100 & 60 & 78,9 \\
\hline $\begin{array}{l}\text { 02. É importante massagear as proeminências ósseas se estiverem } \\
\text { hiperemiadas. (F) }\end{array}$ & 4 & 44,4 & 26 & 34,2 \\
\hline $\begin{array}{l}\text { 03. As pessoas que ficam restritas ao leito devem ser reposicionadas } \\
\text { a cada } 3 \text { horas. }(F)\end{array}$ & 8 & 88,9 & 32 & 42,1 \\
\hline $\begin{array}{l}\text { 04. Uma escala de horários para mudança de decúbito deveria ser } \\
\text { escrita para cada paciente. (V) }\end{array}$ & 8 & 88,9 & 56 & 73,7 \\
\hline 05. Protetores como luvas d'água aliviam a pressão nos calcâneos. (F) & 6 & 66,7 & 18 & 23,7 \\
\hline $\begin{array}{l}\text { 06. A cabeceira da cama deve ser mantida em elevação até } 30^{\circ} \text {, } \\
\text { consistente com as condições. (V) }\end{array}$ & 6 & 66,7 & 49 & 64,5 \\
\hline $\begin{array}{l}\text { 07. Uma pessoa que não pode se movimentar deve ser reposicionada } \\
\text { enquanto sentada a cada } 2 \text { horas. }(F)\end{array}$ & 3 & 33,3 & 50 & 65,8 \\
\hline 08. A pele deve permanecer limpa e seca. (V) & 9 & 100 & 70 & 92,1 \\
\hline $\begin{array}{l}\text { 09. Medidas de prevenção não necessitam ser usadas para prevenir } \\
\text { novas lesões, quando o paciente já possui úlcera por pressão. (F) }\end{array}$ & 8 & 88,9 & 66 & 86,8 \\
\hline $\begin{array}{l}\text { 10. Lençóis móveis ou forros devem ser usados para transferir ou } \\
\text { movimentar o paciente. }(\mathrm{V})\end{array}$ & 9 & 100 & 68 & 89,5 \\
\hline $\begin{array}{l}\text { 11. As proeminências ósseas podem ficar em contato direto uma com } \\
\text { a outra. (F) }\end{array}$ & 9 & 100 & 74 & 97,4 \\
\hline 12. A pele exposta à umidade danifica-se mais facilmente. (V) & 9 & 100 & 68 & 89,5 \\
\hline $\begin{array}{l}\text { 13. Todo cuidado administrado para prevenir ou tratar úlceras por } \\
\text { pressão não precisa ser documentado. (F) }\end{array}$ & 9 & 100 & 66 & 86,8 \\
\hline 14. A fricção pode ocorrer ao movimentar uma pessoa na cama. (V) & 7 & 77,8 & 71 & 93,4 \\
\hline $\begin{array}{l}\text { 15. Para as pessoas que tem incontinência, a limpeza da pele deve } \\
\text { ocorrer no momento que se sujar e nos intervalos de rotina. (V) }\end{array}$ & 8 & 88,9 & 61 & 80,3 \\
\hline $\begin{array}{l}\text { 16. O estágio I da úlcera por pressão é definido como pele intacta, } \\
\text { com hiperemia de uma área localizada, a qual não apresenta } \\
\text { embranquecimento visível ou a com diferença da área ao redor. (V) }\end{array}$ & 8 & 88,9 & 58 & 76,3 \\
\hline $\begin{array}{l}\text { 17. Uma região da pele com cicatriz da úlcera por pressão poderá ser } \\
\text { lesada mais rapidamente do que a pele íntegra. (V) }\end{array}$ & 9 & 100 & 65 & 85,5 \\
\hline $\begin{array}{l}\text { 18. Uma bolha na região do calcâneo não deve ser motivo de } \\
\text { preocupação. }(F)\end{array}$ & 8 & 88,9 & 70 & 92,1 \\
\hline $\begin{array}{l}\text { 19. As úlceras por pressão de estágio II podem ser extremamente } \\
\text { doloridas em decorrência da exposição das terminações nervosas. (V) }\end{array}$ & 7 & 77,8 & 68 & 89,5 \\
\hline
\end{tabular}

de enfermagem e 136 enfermeiros. Também foi avaliada a faixa etária dos participantes, a maior parte dos profissionais (36,3\%) estava na faixa dos 30-40 anos. Os auxiliares/técnicos de enfermagem com a média de 38,5 anos e os enfermeiros com 37,8 anos. Quanto ao sexo, observou-se maior frequência de mulheres $(85,3 \%)$ nos dois grupos profissionais. Os auxiliares/técnicos de enfermagem tinham menor tempo de atuação profissional (média 11,8 anos) do que os enfermeiros (média 12,1 anos) ${ }^{(2)}$.

Tal situação pode ser justificada pelo fato de a enfermagem ser uma profissão majoritariamente feminina e a proporção de técnicos ser maior que a de enfermeiros, em virtude da maior demanda em ambiente hospitalar por esse profissional. E o menor tempo de serviço pode estar relacionado à menor faixa etária das amostras. Em adição, o maior tempo de serviço e a maior idade foram variáveis 
Tabela 3 - Análise bivariada dos resultados obtidos no teste de Pieper e características sóciodemográficas. Montes Claros, MG, Brasil, 2013

\begin{tabular}{|c|c|c|c|c|c|}
\hline \multirow[t]{2}{*}{ Variáveis } & \multicolumn{2}{|c|}{ Enfermeiro } & \multicolumn{2}{|c|}{$\begin{array}{c}\text { Técnico de } \\
\text { Enfermagem }\end{array}$} & \multirow[t]{2}{*}{ Valor $\mathbf{P}$} \\
\hline & $\mathbf{n}$ & $\%$ & $\mathbf{n}$ & $\%$ & \\
\hline \multicolumn{6}{|l|}{ Sexo } \\
\hline Feminino & 6 & 7,1 & 59 & 69,4 & 0,35 \\
\hline Masculino & 3 & 3,5 & 17 & 20 & \\
\hline \multicolumn{6}{|l|}{ Idade } \\
\hline Até 30 anos & 7 & 8,2 & 30 & 35,3 & 0,03 \\
\hline Mais de 30 anos & 2 & 2,4 & 46 & 54,1 & \\
\hline \multicolumn{6}{|l|}{ Tempo de Serviço } \\
\hline Até 5 anos & 5 & 5,9 & 10 & 11,8 & 0 \\
\hline Mais de 5 anos & 4 & 4,7 & 66 & 77,6 & \\
\hline \multicolumn{6}{|c|}{ 1. Fatores de risco para UP } \\
\hline Conhece (acerto) & 9 & 10,6 & 60 & 70,6 & 0,14 \\
\hline Desconhece (erro) & 0 & 0 & 16 & 18,8 & \\
\hline \multicolumn{6}{|c|}{ 2. Massagem em proeminências óssea hiperemiada } \\
\hline Inadequada (acerto) & 4 & 4,7 & 26 & 30,6 & \\
\hline Adequada (erro) & 5 & 5,9 & 50 & 58,8 & 0,4 \\
\hline \multicolumn{6}{|c|}{ 3. Alternância de decúbito de $3 / 3 \mathrm{~h}$} \\
\hline Inadequado (acerto) & 8 & 9,4 & 32 & 37,7 & 0 \\
\hline Adequado (erro) & 1 & 1,2 & 44 & 51,8 & \\
\hline \multicolumn{6}{|c|}{ 4. Alternância de decúbito específico para cada paciente } \\
\hline Adequado (acerto) & 8 & 9,4 & 32 & 37,6 & 0 \\
\hline Inadequado (erro) & 1 & 1,2 & 44 & 51,8 & \\
\hline \multicolumn{6}{|c|}{ 5. Uso de luvas para proteção do calcâneo } \\
\hline Inadequado (acerto) & 6 & 7,1 & 18 & 21,2 & 0 \\
\hline Adequado (erro) & 3 & 3,5 & 58 & 68,2 & \\
\hline \multicolumn{6}{|c|}{ 6. Cabeceira deve ser mantida a $30^{\circ}$} \\
\hline Adequado (acerto) & 6 & 7,1 & 49 & 57,6 & \\
\hline Inadequado (erro) & 3 & 3,5 & 27 & 31,8 & 0,6 \\
\hline \multicolumn{6}{|c|}{ 7. Acamados devem ser movimentados sentados a cada $2 \mathrm{~h}$} \\
\hline Inadequado (acerto) & 3 & 3,5 & 50 & 58,8 & 0,05 \\
\hline Adequado (erro) & 6 & 7,1 & 26 & 30,6 & \\
\hline \multicolumn{6}{|c|}{ 8. A pele deve permanecer limpa e seca } \\
\hline Adequado (acerto) & 9 & 10,6 & 70 & 82,4 & 0,5 \\
\hline Inadequado (erro) & 0 & 0 & 6 & 7,1 & \\
\hline \multicolumn{6}{|c|}{ 9. Paciente que já possui UP dispensa prevenção } \\
\hline Inadequado (acerto) & 8 & 9,4 & 66 & 77,6 & 0,7 \\
\hline Adequado (erro) & 1 & 1,2 & 10 & 11,8 & \\
\hline \multicolumn{6}{|c|}{ 10. Usar lençóis para movimentar pacientes } \\
\hline Adequado (acerto) & 9 & 10,6 & 68 & 80 & 0,4 \\
\hline Inadequado (erro) & 0 & 0 & 8 & 9,4 & \\
\hline \multicolumn{6}{|c|}{ 11. Proeminências ósseas podem ficar em contato direto } \\
\hline Inadequado (acerto) & 9 & 10,6 & 74 & 87,1 & 0,8 \\
\hline Adequado (erro) & 0 & 0 & 2 & 2,4 & \\
\hline
\end{tabular}


Tabela 3 - Análise bivariada dos resultados obtidos no teste de Pieper e características sóciodemográficas. Montes Claros, MG, Brasil, 2013 (continuação)

\begin{tabular}{|c|c|c|c|c|c|}
\hline \multirow[t]{2}{*}{ Variáveis } & \multicolumn{2}{|c|}{ Enfermeiro } & \multicolumn{2}{|c|}{$\begin{array}{l}\text { Técnico de } \\
\text { Enfermagem }\end{array}$} & \multirow[t]{2}{*}{ Valor $\mathbf{P}$} \\
\hline & $\mathrm{n}$ & $\%$ & $\mathbf{n}$ & $\%$ & \\
\hline \multicolumn{6}{|c|}{ 12. Pele úmida danifica-se facilmente } \\
\hline Adequado (acerto) & 9 & 10,6 & 68 & 80 & 0,4 \\
\hline Inadequado (erro) & 0 & 0 & 8 & 9,4 & \\
\hline \multicolumn{6}{|c|}{ 13. Cuidados com UP não precisam ser documentados } \\
\hline Inadequado (acerto) & 9 & 10,6 & 66 & 77,6 & 0,3 \\
\hline Adequado (erro) & 0 & 0 & 10 & 11,8 & \\
\hline \multicolumn{6}{|c|}{ 14. Fricção pode ocorrer ao movimentar pacientes } \\
\hline Adequado (acerto) & 7 & 8,2 & 71 & 83,5 & 0,15 \\
\hline Inadequado (erro) & 2 & 2,4 & 5 & 5,9 & \\
\hline \multicolumn{6}{|c|}{ 15. Pessoas com incontinência devem ser higienizados constantemente } \\
\hline Adequado (acerto) & 8 & 9,4 & 61 & 71,8 & 0,46 \\
\hline Inadequado (erro) & 1 & 1,2 & 15 & 17,6 & \\
\hline \multicolumn{6}{|c|}{ 16. Definição de UP estágio I } \\
\hline Adequado (acerto) & 8 & 9,4 & 58 & 68,2 & 0,35 \\
\hline Inadequado (erro) & 1 & 1,2 & 18 & 21,2 & \\
\hline \multicolumn{6}{|c|}{ 17. UP cicatrizada lesa mais facilmente } \\
\hline Adequado (acerto) & 9 & 10,6 & 65 & 76,5 & 0,3 \\
\hline Inadequado (erro) & 0 & 0 & 11 & 12,9 & \\
\hline \multicolumn{6}{|c|}{ 18. Bolhas no calcâneo não é risco } \\
\hline Inadequado (acerto) & 8 & 9,4 & 70 & 82,4 & 0,55 \\
\hline Adequado (erro) & 1 & 1,2 & 6 & 7,1 & \\
\hline \multicolumn{6}{|c|}{ 19. Definição de UP estágio II } \\
\hline Adequado (acerto) & 7 & 8,2 & 68 & 80 & 0,3 \\
\hline Inadequado (erro) & 2 & 2,4 & 8 & 9,4 & \\
\hline
\end{tabular}

significantes, o que sugere maior experiência e contato por maior tempo com situações que ocasionam o referido agravo, viabilizando maior conhecimento.

Diferentemente deste, em outro trabalho, observou-se que a porcentagem de acertos no teste dos auxiliares/técnicos de enfermagem diminuiu com o tempo de formação profissional $(p=0,009)$, assim como com o tempo de serviço $(p=0,049)$. Entretanto, no grupo dos enfermeiros, a correlação encontrada entre a porcentagem de acertos e essas variáveis não foi estatisticamente significante $^{(2)}$.

Estudo similar realizado em um hospital universitário do interior paulista demonstrou que, com relação às 33 questões do teste, em 16 itens os enfermeiros obtiveram $100 \%$ de acertos e em 10 obtiveram $85,7 \%$ de acertos. O item que obteve menor índice de acertos $(28,6 \%)$ foi a afirmação referente à necessidade de reposicionamento a cada 2 horas do paciente em cadeira de rodas (que não consegue se movimentar sem ajuda), o que evidencia desconhecimento em relação ao tempo ideal para alívio das áreas de pressão. $\mathrm{O}$ recomendado é que a permanência em poltrona/ cadeiras de rodas não deve exceder duas horas, durante as quais se devem alternar os pontos de pressão pelo menos a cada hora ${ }^{(4)}$.

O conhecimento das medidas de prevenção e das características da úlcera por pressão deve fazer parte do rol de conhecimento de todos os profissionais da área de enfermagem, já que são agravos evitáveis. Assim, o desconhecimento apresentado no estudo pode estar associado à falta de capacitação e educação permanente da equipe de enfermagem quanto à temática.

A afirmação número dois, que trata da contraindicação de massagem nas proeminências ósseas hiperemiadas, apresentou baixo índice de acertos em ambas as categorias dos profissionais de enfermagem. No estudo realizado 40 profissionais da equipe de enfermagem que 
atuam no centro de terapia intensiva, na cidade de João Pessoa-PB, resultado semelhante foi obtido, em que apenas $36,9 \%$ profissionais que participaram da pesquisa acertaram a questão ${ }^{(2)}$.

Vale ressaltar que essa é uma recomendação de nível de evidência B, conforme o teste de Pieper, já que em proeminências ósseas hiperemiadas há presença de inflamação aguda, na qual existe possibilidade de haver vasos sanguíneos danificados ou pele frágil, o que faz deste um fator importante na prevenção das úlceras ${ }^{(9)}$.

Em uma investigação que avaliou o conhecimento sobre prevenção e tratamento de úlcera por pressão em hospital de Minas Gerais, as medidas preventivas mais citadas foram a mudança de decúbito, em que $100 \%$ afirmaram conhecer e praticar. Cinco profissionais (33,3\%) relataram manter o paciente seco/realizar higiene do paciente. A realização de massagem foi citada por três dos profissionais (20\%); um participante citou a elevação da cabeceira em $30^{\circ(10)}$.

Ao se analisar a média global de acertos, nesta pesquisa, percebeu-se $84,21 \%$ de acertos para os enfermeiros e de $68,42 \%$ para os técnicos/ auxiliares de enfermagem. Diferença considerável na porcentagem de acertos entre as duas categorias foi observada $(15,79 \%)$. Os escores das duas categorias variaram de 10 a 19 pontos. $O$ resultado deste estudo demonstra déficit de conhecimento dos participantes da pesquisa. Para o conhecimento ser considerado adequado, esperava-se que os participantes acertassem $90 \%$ ou mais dos itens no teste de Pieper.

Nos Estados Unidos, uma investigação com enfermeiros da zona urbana e rural de Montana utilizou a versão preliminar do teste de conhecimento de Pieper, e a porcentagem média de acertos obtida no teste foi de $78 \%$ 12). Já pesquisa realizada na Espanha, utilizando questionário baseado em diretrizes nacionais, com 37 questões, também avaliou o nível de conhecimento dos enfermeiros e técnicos sobre prevenção das úlceras. Verificou-se índice de acertos de $78 \%$, sendo que para as medidas preventivas o índice de acertos foi de $79,1 \%{ }^{(13)}$.

Os enfermeiros e técnicos, majoritariamente, apresentam deficiência no conhecimento sobre as recomendações de prevenção de UPs. Um estudo realizado para avaliar o impacto de uma intervenção educativa, utilizando o teste de conhecimento de Pieper adaptado, identificou que, na fase pré-intervenção, os enfermeiros obtiveram $86,4 \%$ de acertos, porém, nenhum profissional participou da avaliação posterior. Os auxiliares e técnicos de enfermagem obtiveram $74,3 \%$ de acertos, na fase pré-intervenção, e $81,2 \%$, na fase pós-intervenção, realizada 20 semanas após o curso. Portanto, para o grupo profissional em questão, a educação em serviço colaborou para melhoria nos resultados do teste ${ }^{(4)}$.

Embora seja um indicador de qualidade negativo dos serviços de saúde, a úlcera por pressão ainda é um problema subestimado pelos profissionais. Persiste a ocorrência frequente em pacientes internados, caracterizando um cenário que mostra a necessidade de medidas urgentes para reverter a situação. $O$ déficit de conhecimento, aliado ao uso de práticas inadequadas pelos profissionais, contribui para a manutenção da realidade. A prevenção da ocorrência da úlcera requer melhor compreensão da equipe de enfermagem sobre todos os aspectos que envolvem o seu desenvolvimento, assim como atitudes para um cuidar ético com a adoção das práticas recomendadas, incluindo a busca de recursos adequados. Diversas estratégias podem ser utilizadas para melhorar o nível de conhecimento dos profissionais, mas se torna necessário identificar as barreiras pessoais e institucionais que dificultam o atendimento a esta meta ${ }^{(4,8)}$.

O conhecimento da equipe de enfermagem sobre úlcera por pressão é tão importante quanto a prevenção do risco para desenvolvê-la. Planejar intervenções efetivas, a partir da criação de protocolos de prevenção e capacitação adequada se torna importante, na busca incansável e contínua na melhoria da qualidade da assistência de enfermagem, desempenhando o cuidado com responsabilidade e autonomia, sem implicar a vida profissional e sem agravar o estado clínico do paciente ${ }^{(14)}$, o que certamente promoverá maior segurança do paciente.

Assim, a utilização de escalas, como a Escala de Braden de avaliação de risco para úlcera por pressão, requer pouca habilidade do profissional enfermeiro, embora demande mais tempo na assistência para sua avaliação e acompanhamento. Contudo, é de extrema importância para o prognóstico, já que a identificação dos pacientes em risco permite a implementação de medidas preventivas precocemente, o que pode reduzir pela metade a incidência das UPs ${ }^{(15-16)}$. Ademais, é de suma importância a implementação da Sistematização da Assistência de Enfermagem (SAE) que pode levantar questionamentos para o risco e elaboração de planos de cuidados individualizados para pacientes com fatores 
de risco para o desenvolvimento da úlcera por pressão $^{(16-18)}$.

O presente trabalho tem como limitações o fato de que os indivíduos alocados se restringiram a um cenário limitado, o que pode afetar o poder de generalização dos resultados. O desenho foi transversal, que impede declarações de causa e efeito. Nesse sentido, tais limitações e o contexto relativo ao cenário local e às suas singularidades podem ser objeto de outros estudos.

\section{CONCLUSÃO}

No presente estudo, foi possível evidenciar que a maioria dos profissionais da enfermagem demonstrou conhecimento insatisfatório sobre a prevenção das UPs. Isso foi ainda mais presente entre os técnicos de enfermagem. Houve diferença significativa de conhecimento entre as duas categorias: os enfermeiros mostraram maior conhecimento no teste, ao inverso dos técnicos de enfermagem. Esses achados sinalizam uma realidade preocupante, sobretudo, ao se considerar que a prevenção das UPs está diretamente vinculada à segurança do paciente $\mathrm{e}$ à qualidade da assistência.

Ainda, ter maior idade e mais tempo de atuação são características que contribuíram para um maior conhecimento entre os profissionais pesquisados. Sendo assim, os profissionais que possuem essas características podem contribuir para a reversão da realidade identificada, no sentido de orientar os seus colegas. Tal apoio também seria pertinente para questões sobre massagem das proeminências ósseas e uso de protetores como luvas d'água, itens com grande carência de conhecimento.

Assim, a deficiência de conhecimentos sobre o assunto tratado é evidente. Nesse sentido, ressalta-se a necessidade da educação continuada e permanente, sobre as medidas preventivas das UPs, bem como as questões relativas ao estadiamento das mesmas. A educação permanente, acompanhada de uma contínua avaliação do conhecimento, com os profissionais de enfermagem da instituição, incorporaria novos conhecimentos, tecnologias e opções disponíveis para utilização em suas práticas; bem como clarificação da responsabilidade frente à questão da prevenção das UPs.

\section{REFERÊNCIAS}

1. Wada A, Teixeira Neto N, Ferreira MC. Úlceras por pressão. Rev Med. [Internet] 2010; 89(3) [acesso em
11 nov 2014]. Disponível: http://www.revistas.usp.br/ revistadc/article/view/46293/49949

2. Miyazaki MY, Caliri MHL, Santos CB. Conhecimento dos profissionais de enfermagem sobre prevenção da úlcera por pressão. Rev Latino-Am Enfermagem. [Internet] 2010; 18(6) [acesso em 19 nov 2014]. Disponível: http://www.scielo.br/pdf/rlae/v18n6/pt_22

3. Albuquerque AM, Souza MA, Torres VSF, Porto VA, Soares MJGO, Torquato IMB. Assessment and prevention of pressure ulcer by nurses from intensive care: knowledge and practice. Rev Enferm UFPE On line. [Internet] 2014;8(2) [acesso em 22 nov 2014]. Disponível: http://www.revista.ufpe.br/revistaenfermagem/index. php/revista/article/view/4688/pdf_4510

4. Fernandes LM, Caliri MHL, Haas VJ. The effect of educative interventions on the pressure ulcer prevention knowledge of nursing professionals. Acta Paul Enferm. [Internet] 2008; 21(2) [acesso em 14 nov 2014]. Disponível: http://dx.doi.org/10.1590/S010321002008000200012

5. Ferreira AM, Rigotti MA, Pena SB, Silva Paula D, Ramos IB, Sasaki VDM. Conhecimento e prática de acadêmicos de enfermagem sobre cuidados com portadores de feridas. Esc Anna Nery. [Internet] 2013; 17(2) [acesso em 14 nov 2014]. Disponível: http://dx.doi. org/10.1590/S1414-81452013000200002

6. Chayamiti EMPC, Caliri MHL. Pressure ulcer in patients under home care. Acta Paul Enferm. [Internet] 2010; 23(1) [acesso em 14 nov 2014]. Disponível: http:// dx.doi.org/10.1590/S0103-21002010000100005

7. Morita ABPS, Poveda VB, Santos MJ, Marcelino AL. Conhecimento dos enfermeiros acerca dos instrumentos de avaliação de risco para úlcera por pressão. REENVAP. [Internet] 2012; 02 [acesso em 19 nov 2014]. Disponível: http://publicacoes.fatea.br/ index.php/reenvap/article/viewFile/555/386

8. Trueman P, Whitehead SJ. The economics of pressure relieving surfaces: an illustrative case study of the impact of high-specification surfaces on hospital finances. Int Wound J. 2010; 7(1):48-54.

9. Smith ME, Totten A, Hickam DH, Fu R, Wasson N, Rahman B, et al. Pressure ulcer treatment strategies: a systematic comparative effectiveness review. Ann Intern Med. 2013; 159(1):39-50.

10. Martins AD, Soares FFR. Conhecimento sobre prevenção e tratamento de úlceras por pressão entre trabalhadores de enfermagem em um hospital de Minas Gerais. Cogitare enferm. [Internet] 2008; 13(1) [acesso em 19 nov 2014]. Disponível: http://ojs.c3sl. ufpr.br/ojs/index.php/cogitare/article/view/11956/8437

11. Zulkowiski K, Avello EA, Wexler S. Certification and education: do they affect pressure ulcer knowledge in 
12. Blanc G, Meier MJ, Stocco JGD, Roehrs H, Crozeta $\mathrm{K}$, Barbosa DA. Effectiveness of enteral nutritional therapy in the healing process of pressure ulcers: a systematic review. Rev Esc Enferm USP. [Internet] 2015; 49(1) [acesso em 25 jul 2015]. Disponível: http://dx.doi. org/10.1590/S0080-623420150000100020

13. Pancorbo-Hidalgo PL, García-Fernández FP, LópezMedina IM, López-Ortega J. Pressure ulcer care in Spain: nurses' knowledge and clinical practice. J Adv Nurs. 2007; 58(4):327-38.

14. Anselmi ML, Peduzzi M, França Junior I. Incidence of pressure ulcer and nursing interventions. Acta Paul Enferm. [Internet] 2009; 22(3) [acesso em 04 jan 2014]. Disponível: http://dx.doi.org/10.1590/S010321002009000300004

15. Borghardt AT, Prado TN, Araújo TM, Rogenski NMB, Bringuente MEO. Evaluation of the pressure ulcers risk scales with critically ill patients: a prospective cohort study. Rev Latino-Am Enfermagem. [Internet] 2015; 23(1) [acesso em 24 jul 2015]. Disponível: http://dx.doi. org/10.1590/0104-1169.0144.2521

16. Araújo TM, Araújo MFM, Caetano JA, Galvão MTG, Damasceno MMC. Diagnósticos de enfermagem para pacientes em risco de desenvolver úlcera por pressão. Rev Bras Enferm. [Internet] 2011; 64(4) [acesso em 04 nov 2014]. Disponível: http://dx.doi.org/10.1590/S003471672011000400007

17. Van Hecke A, Beeckman D, Grypdonck $M$, Meuleneire F, Hermie L, Verhaeghe S. Knowledge deficits and information-seeking behavior in leg ulcer patients: an exploratory qualitative study. J Wound Ostomy Continence Nurs. 2013; 40(4):381-7.

18. Silva DC, Budó MLD, Schimith MD, Ecco L, Costa IKF, Torres GV. Experiences constructed in the process of living with a venous ulcer. Cogitare enferm. [Internet] 2015; 20(1) [acesso em 24 jul 2015]. Disponível:

http://ojs.c3sl.ufpr.br/ojs/index.php/cogitare/article/ view/37784/24830 